\title{
Hydro-chemo-mechanical processes in soil samples: monitoring through electrical resistivity tomography
}

\author{
C. Comina ${ }^{1}$, R. M. Cosentini ${ }^{2}$, G. Della Vecchia ${ }^{2}$, S. Foti ${ }^{2, a}$ and G. Musso ${ }^{2}$ \\ ${ }^{1}$ Università di Torino, Department of Earth Science, via Valperga Caluso, 3510125 Torino, Italy \\ ${ }^{2}$ Politecnico di Torino, Department of Structural and Geotechnical Engineering, corso Duca degli \\ Abruzzi 24, 10129 Torino, Italy
}

\begin{abstract}
The electrical conductivity of a soil can be related to the electrical conductivity of its solid and fluid constituents by means of theoretical and empirical relationships, taking into account the effect of porosity, saturation degree, fabric and clay content. Hence monitoring the evolution of the electrical conductivity inside a soil sample can provide useful information concerning the progress of hydro-chemomechanical processes and the subsequent effects on both soil skeleton and pore water. With this aim a laboratory apparatus for 3D electrical resistivity tomography has been recently developed. Some applications of this apparatus to the monitoring of different geotechnical processes in which the knowledge of the water content and of ionic concentration is particularly relevant are presented.
\end{abstract}

\section{Introduction}

Electrical Resistivity Tomography (ERT) is a technique of common application in geophysical site investigation, generally adopted for the reconstruction of spatial distribution of the electrical conductivity. In situ applications of ERT mainly regard the reconstruction of geological horizons and the monitoring of soil water and contaminants flow. Nonetheless, interesting results have been obtained also at the laboratory scale, where ERT can be applied as a subsidiary imaging tool for the characterization of soil samples. On basis of constitutive relationships, it possible as well to obtain important information about the state of a porous sample: the influence of porosity, fabric, distribution of interstitial fluids and conductivity of constituent phases on the overall electrical conductivity is well documented in the literature (e.g. [1]). Standard electric measurements at the laboratory scale have been used for instance to estimate the diffusion of salt in soil samples [2], to detect clay sample anisotropy [3] and to monitor sedimentation processes [4].

The advantage of ERT with respect to standard electric measurements consists in the possibility to estimate also the spatial variability of the electrical conductivity distribution inside the specimen, using measurements entirely performed from the external boundaries. The local distribution of electrical conductivity is reconstructed by a sufficiently high number of independent measurements and the subsequent solution of an inverse problem. Two dimensional ERT has already been applied with encouraging results by several authors to visualize liquefaction [5], to individuate

\footnotetext{
a e-mail : sebastiano.foti@polito.it
} 


\section{EPJ Web of Conferences}

different kinds of heterogeneities [6] and to monitor diffusion processes of saline solution [7, 8,9$]$. The passage to 3D reconstruction, even if it complicates both the inverse problem solution and the measurement sequence, appears however mandatory for a complete comprehension of phenomena involving the whole sample.

\section{Instrumentation}

All the tests described in the present work were run in the advanced oedometer cell illustrated in [10]. This device has been specifically designed in order to perform 3D ERT and to measure the velocity of compressional (P) and shear (S) seismic waves. Conventional hydro-mechanical external measurements (inflow/outflow of water and vertical displacement) and controls (imposed vertical load and prevented radial displacement and fluid flux) are allowed. The cell (shown in Figure 1a) has a diameter of $130 \mathrm{~mm}$ and it can host samples with maximum height of $60 \mathrm{~mm}$. In order to perform ERT measurements, 42 electrodes are located on the internal boundary of the cell, 16 equally spaced on the sidewall and 13 on each base (Figure 2a). The soil sample is completely insulated from the confining stainless steel of the cell by means of a high resistivity material that prevents short circuiting. Particular care has been posed also in the insulation of water flow paths. For instance, water drainage at the top and at the bottom of the sample is allowed by concentric rings of the insulating material, having a few micron tolerance between them (Figure 2a). The equivalent hydraulic conductivity of the bases, averaged on their full section, has been evaluated to be approximately $6 \cdot 10^{-6} \mathrm{~m} / \mathrm{s}$.

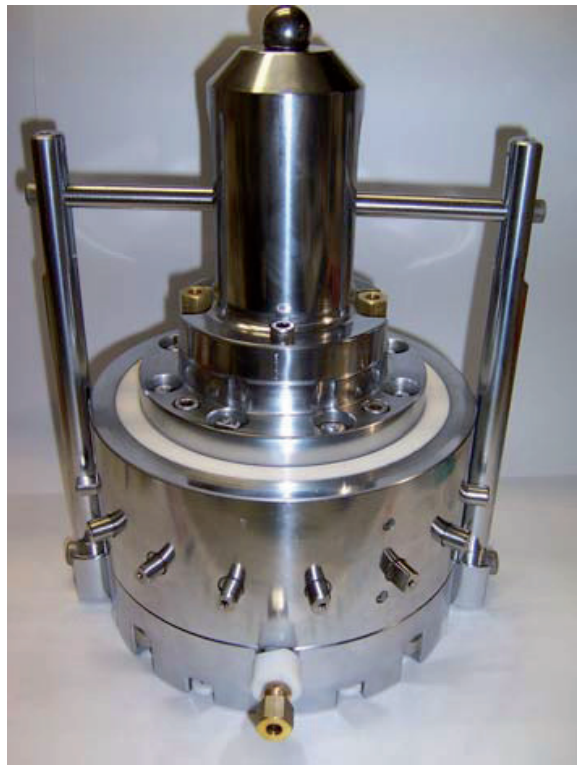

Fig. 1. Oedometer ERT cell used in the investigation.

The Complex Impedance Tomograph used for injecting currents and acquiring potentials, constructed by Iridium Italy (Figure 2b), has a resolution of 16 bit and can perform approximately 200 acquisition per second at an operating frequency of $1 \mathrm{kHz}$. Electrical measurements are performed on basis of a four point measurements scheme: electrodes placed on the sidewall and on the bases are used alternatively to inject current and to measure the electric potential difference existing between them. 
In order to obtain a complete 3D characterization of the specimen, measurements protocol combines different acquisition schemes:

- horizontal measurements: electrodes injecting the electrical current and those measuring potential variations are on the sidewall;

- vertical measurements: electrodes injecting the electrical current and those measuring potential variations are on the bases;

- mixed measurements: electrodes injecting the electrical current are on the sidewall and those measuring potential variations are on the bases.
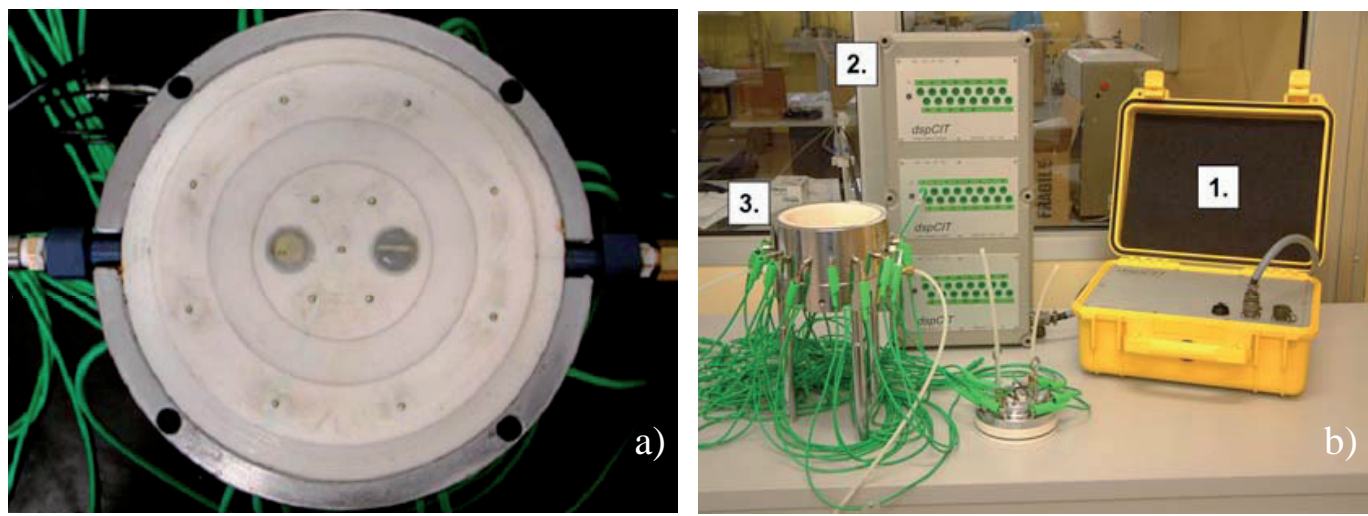

Fig. 2. a) Drainage system at the base of the cell and base electrodes position, b) Measurement setup: 1. Complex impedance tomograph; 2 . Multiplexers and connection to the cell; 3 . Oedometer ERT cell.

With a sufficiently large number of independent electrical measurements, the spatial distribution of the electrical conductivity within the sample is estimated by the means of a reconstruction procedure, based on a least-squares algorithm with a Tikhnov regularization inversion technique (see Borsic et al., 2005, for further details). The forward algorithm implemented within the software evaluates the current flow generated from the electrodes, modeled using their real dimension. The mesh used for the solution of the forward problem is reported in Figure 3, together with a detail showing the refinement around the electrodes.

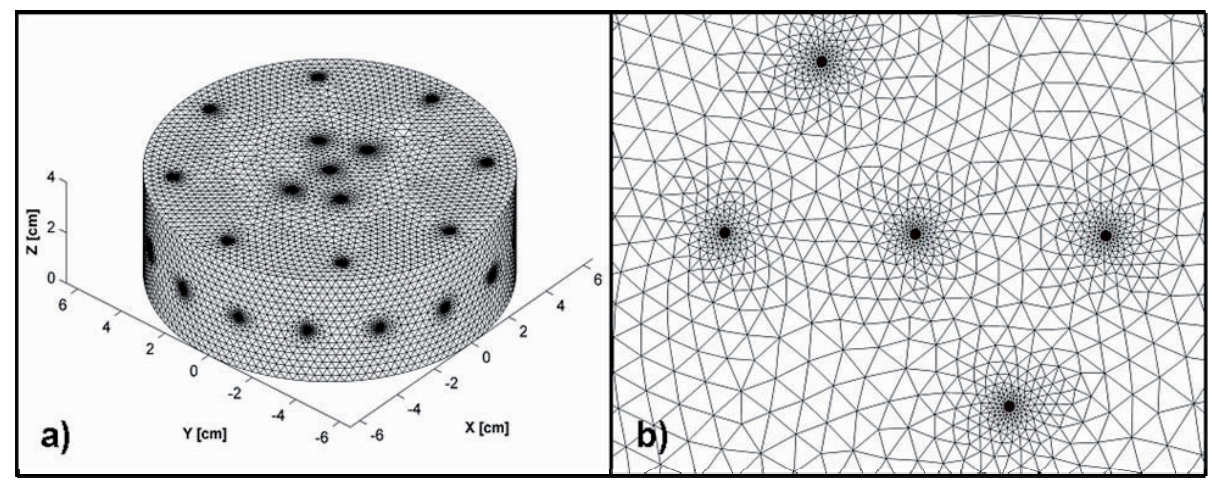

Fig. 3. FEM mesh: a) global view; b) detail showing refinements around the electrodes. 


\section{Monitoring of transient phenomena in soil samples}

The experimental tests presented are devoted at exploring the potentiality of ERT in monitor water content and salt concentration variations in soil specimens. These variations can indeed influence the mechanical response of soil samples, so that imaging their distribution in geotechnical testing can actually improve the interpretation of tests aimed at investigating fundamental soil behaviour. Some results concerning chemical diffusion, mechanical consolidation and water saturation changes are discussed in the following sections.

\subsection{Three-dimensional chemical diffusion}

Due to the variation in electrical conductivity of interstitial water caused by variation in salt concentration, ERT can be used to determinate the distribution of solute during chemical diffusion processes. The test here described was performed on a sample of Ticino sand, an almost monogranular quartz sand with mean particle size of $0.5 \mathrm{~mm}$, initially saturated with distilled water. Chemical diffusion was induced by placing a localized saline source $(\mathrm{NaCl}$ grains) at the upper boundary of the cell. The use of pure $\mathrm{NaCl}$ assures a constant concentration condition, in which the concentration is equal to salt solubility. Due to the very large resistivity of quartz minerals and to the absence of porosity variations during the test, any local change of the electrical conductivity can be related to a corresponding change in the pore fluid properties (in this case chemical concentration).

Tomography data have been acquired at different time intervals from the beginning of the test. Figure $4 \mathrm{a}$ shows the reconstructed distribution of the electrical conductivity after 75 minutes from the beginning of the process. The higher conductivity area at the top of the specimen evidences the increased chemical concentration, which is shown to progress as an hemispherical front.

The following test was aimed at evidencing the capability of detecting retardation effects, due to adsorption and higher soil tortuosity. A kaolinite cylinder $1 \mathrm{~cm}$ thick was then placed in the middle of a similar Ticino sand sample, subjected to the same boundary condition. The reconstructed image referring to the $75^{\text {th }}$ minute (Figure $4 \mathrm{~b}$ ) suggests a shallower penetration of salt, together with a lateral percolation due to a loose contact of the kaolinite with the cell walls.
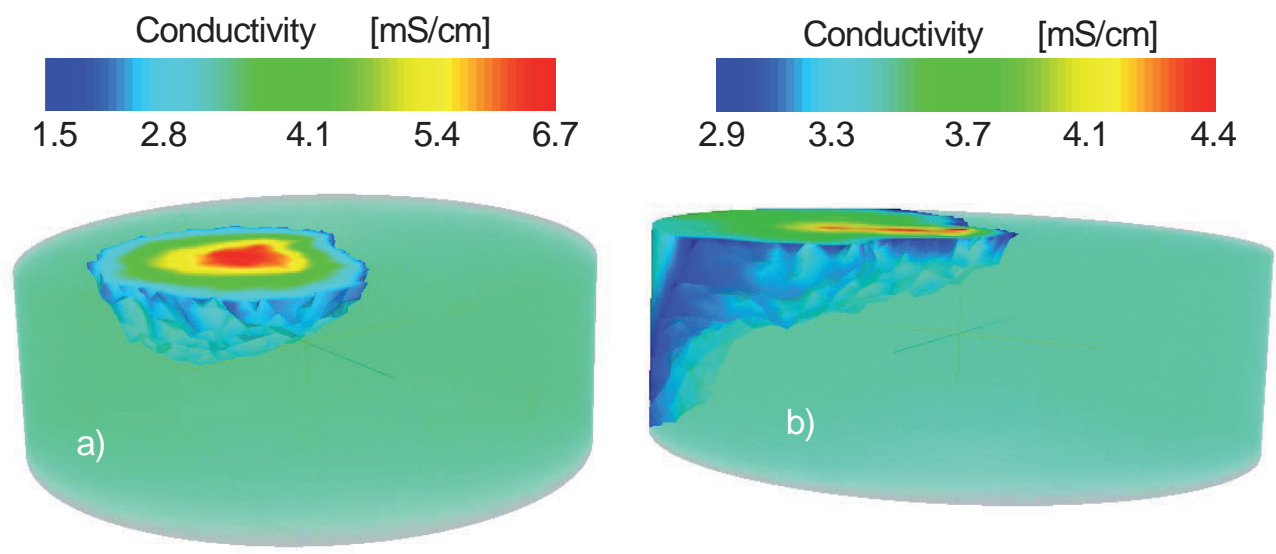

Fig. 4. Diffusion test: ERT reconstruction after 75 minutes from the beginning of the diffusion: a) homogeneous sample; b) sample with kaolinite intercalation. 


\subsection{Mechanical consolidation processes}

In another set of tests, the attention was driven to the possibility of using ERT to monitor variations in soil porosity due to mechanical loads, i.e. monitoring consolidation processes in oedometer conditions. The most used expression relating the electrical conductivity of a porous medium $\sigma$ to the porosity $n$ is the so called Archie's law [11], that holds under the assumption of non-conductive solid phase. If the porous space is fully saturated with water, its expression is:

$$
\sigma=\sigma_{w} n^{m}
$$

where $\sigma_{w}$ is the electrical conductivity of the water phase and $m$ an exponent that accounts for the effect of tortuosity.

An oedometer test was performed on a preconsolidated kaolinite homogeneous sample, saturated with tap water. The limited specific surface and cation exchange capacity of kaolinite leads to a very moderate surface conductivity, so that eq. 1 can be used to interpret the test results and the electrical conductivity evolution can be solely linked to changes in porosity. Experimental data presented in details in [8], confirm a progressive average reduction of conductivity during sample compression and an increase during swelling, which can be reasonably interpreted in light of equation 1 .

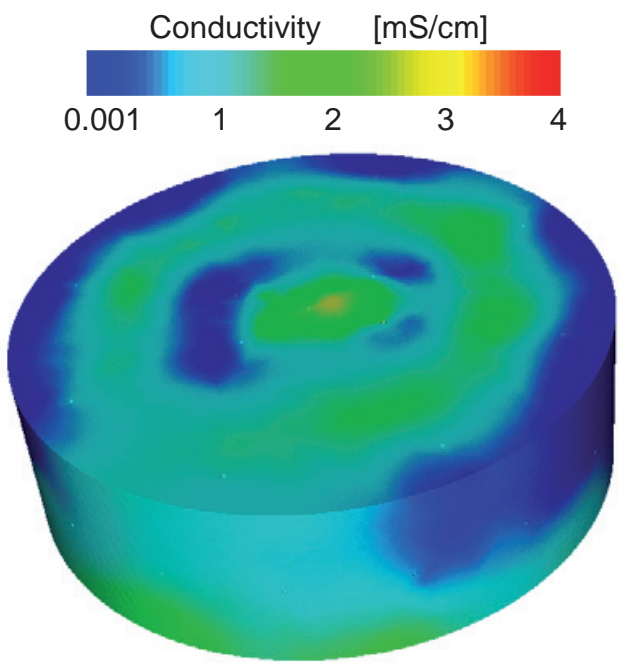

Fig. 5. Mechanical test: differential image of tomography reconstruction immediately before and after the application of a load increment.

The advantage of ERT is however to monitor the local distribution of conductivity decrease or increase. A differential image obtained immediately after the application of a load step is presented in this respect in Figure 5. The figure shows the differences between the local electrical conductivity immediately before and after imposing the load. The higher values represent higher differences in local conductivities, indicating areas where consolidation increased further in the time span considered. The unusual distribution encountered is dictated by the hydraulic boundary conditions of the cell, allowing water to flow only from the three localized concentric drainages on the top of the cell (see Figure 2). 


\subsection{Unsaturated soils}

The effect of variation of degree of saturation $S_{r}$ on the electrical conductivity of a porous medium, keeping the assumption of non-conductive solid phase, can be expressed by an extension of Equation 1 :

$$
\sigma=\sigma_{w} n^{m} S_{r}^{p}
$$

where $p$ is an empirical parameter, whose value can be related to the pore size distribution of the material [12]. For a given soil porosity and fabric, if the electrical conductivity of the pore water is kept constant, a reduction of the water content involves a decrease in conductivity and viceversa.

Experiments have been conducted on homogeneous samples to verify if ERT can be used to indirectly evaluate the degree of saturation, both in stationary and transient conditions. As for stationary conditions, a number of samples was dynamically compacted at the same average porosity $\mathrm{n}=0.4$ and at different values of saturation degree. In order to ensure a constant $\sigma_{w}$, a $0.1 \mathrm{M} \mathrm{KCl}$ water solution was used as liquid phase. Figure 6 shows the tomographic images obtained for degree of saturation ranging from $10 \%$ to $100 \%$. The significant impact of saturation on electrical conductivity can be appreciated.

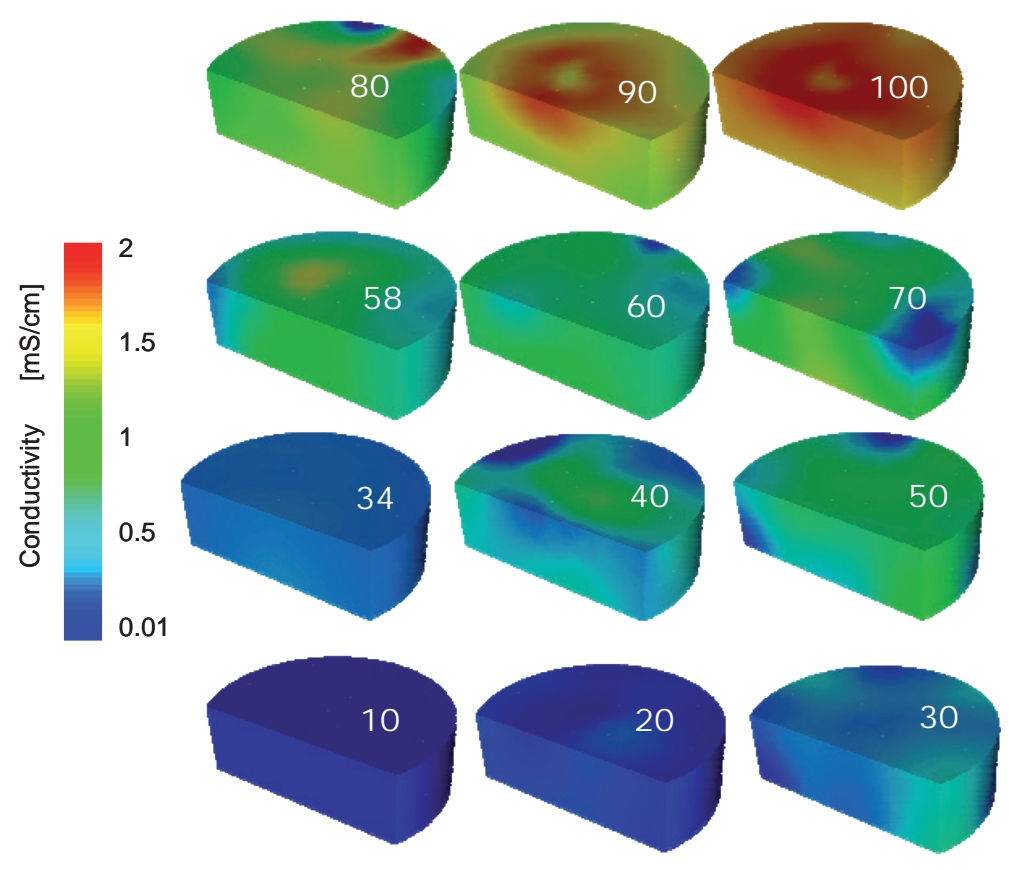

Fig. 6. Unsaturated soil test in stationary conditions: tomographic reconstruction of sand samples at different saturation degrees, overwritten in percentage on the images.

As for transient conditions, the evolution of the local and average water content of a silty sand specimens was monitored. The specimen has been prepared by dynamic compaction at a porosity $n=0.45$ and a degree of saturation $S_{r}=0.2$. The sample has been wetted by the inflow of a water volume of $90 \mathrm{~cm}^{3}$, that occurred in $40 \mathrm{~s}$ by applying a water pressure of $50 \mathrm{kPa}$ at its base. At the end of the wetting stage, the bottom drainage system has been closed and the spatial distribution of the electrical conductivity monitored for 3000 minutes. Collected information has been used for the reconstruction of the conductivity map at selected time steps. 


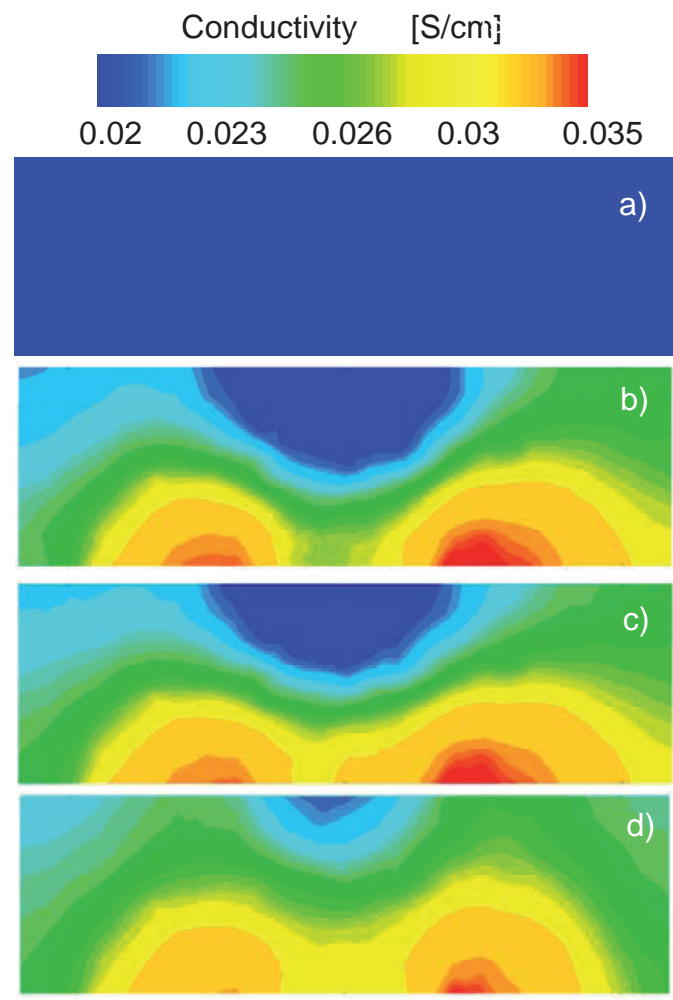

Fig. 7. Unsaturated soil test (non-stationary conditions). Tomographic reconstructions of homogeneization stage: a) $t=0 \mathrm{~min}, \mathrm{~b}) \mathrm{t}=10 \mathrm{~min}, \mathrm{c}) \mathrm{t}=110 \mathrm{~min}, \mathrm{~d}) \mathrm{t}=3000 \mathrm{~min}$.

Figure 7 reports the tomographic reconstructions of a vertical section of the sample obtained at time $\mathrm{t}_{0}=0 \mathrm{~min}$ (before drainage opening), $\mathrm{t}_{1}=10 \mathrm{~min}, \mathrm{t}_{2}=110 \mathrm{~min}$ and $\mathrm{t}_{3}=3000 \mathrm{~min}$. A progressive homogenization of the local water content process, even if not fully ended within the testing interval, is clearly shown. In respect to the starting situation can be indeed noticed the presence of higher conductivity zones (higher water content) from the bottom base; again the influence of the drainage paths can be noticed.

\section{Conclusions}

Experimental tests have been performed in order to check the ability of 3D ERT technique in monitoring transient phenomena in soils samples. The results show that the technique allows for identifying water content changes, evolution of porosity during mechanical consolidation and saline diffusion in laboratory samples by means of the average and local variations of the reconstructed electrical conductivity. Laboratory ERT appears as a useful tool to support in situ measurements for problems involving distribution of water and chemical species in the soil. In particular this technique can furnish laboratory calibrations for site applications and qualitative and quantitative interpretations useful to link electrical measurements to soil characteristics. The next step in the ongoing research is related to the use of ERT reconstructions in back analysis procedures aimed at estimating transport parameters for saturated and unsaturated soils. 


\section{References}

1. J.C. Santamarina, K.A. Klein, M.A Fam, Soils and Waves (John Wiley and Sons, New York, 2001)

2. C.D. Shackelford, M.A. Malusis, M.J. Majeski, R.T. Stern, J. Geotech. and Geoenv. Engrng. 125, 4, 260-270 (1999)

3. W.J. McCarter, J. Blewett, T.M. Chrisp, G. Starrs, Can. Geotech. J., 42, 655-662 (2005)

4. J. Blewett, W.J. McCarter, T.M. Chrisp, G. Starrs, Géotechnique 51, 8, 723-728 (2001)

5. M. Jinguuji, S. Toprak, S. Kunimatsu, Soil Dynamics and Earthquake Engineering, 27, 191-199 (2007)

6. A. Borsic, C. Comina, S. Foti, R. Lancellotta, G. Musso, Géotechnique 55, 7, 539-547 (2005)

7. A. Binley, B. Shaw and S. Henry-Poulter, Meas. Sci. Technol. 7, 384-390 (1996)

8. C. Comina, S. Foti, R. Lancellotta, G. Musso, A. Borsic, Proc. IACMAG2005, vol.2, 27-34 (2005)

9. V.M. Damasceno, D. Fratta, P.J. Bosscher, Can. Geotech. J., 46, 842-854 (2009)

10. C. Comina, S. Foti, G. Musso, E. Romero, Geotech. Test. J., ASTM, 31, 5, 404-412 (2008)

11. G.E. Archie, Trans AIME 146, 54-63 (1942)

12. P. N. Sen, Geophysics, 62, 2 , 415-425 (1997) 